BULLETIN OF THE

AMERICAN MATHEMATICAL SOCIETY

Volume 78, Number 4, July 1972

\title{
HOLOMORPHIC FUNCTIONS ON BALANCED SUBSETS OF A BANACH SPACE
}

\author{
BY RICHARD M. ARON ${ }^{1}$
}

Communicated by Felix Browder, December 29, 1971

1. The study of nuclearly-entire functions between two complex Banach spaces was begun by Nachbin and Gupta in [7] and [8] and has recently been extended by Dineen to entire functions of $\alpha$-holomorphy type $\theta$ in [3] and [4]. In §2, we describe a generalization of Dineen's work to holomorphic functions on balanced open subsets of a Banach space. This generalization is applied in $\S 3$ to characterize the space of holomorphic germs of $\alpha$-holomorphy type $\theta$ over a compact, convex balanced set.

Our notation and terminology will follow that given in [3], [4], and [6]. For convenience, we give the most important terms here. $U$ will always denote an open, balanced nonvoid subset of the complex Banach space. $\mathscr{K}(U)$ will denote the family of compact, convex, balanced subsets of $U$. For simplicity of notation, we will assume that our holomorphic mappings are complex valued; there is no difficulty in extending all results to Banach space valued mappings.

$\theta$ will be a holomorphy type, and $\left(\mathscr{H}_{\theta}(U), \tau_{\omega \theta}\right)$ will be the space of holomorphic functions on $U$ of holomorphy type $\theta$, endowed with the locally convex topology $\tau_{\omega \theta}[6, \mathrm{pp} .34-35,43]$. When $\theta$ is the current holomorphy type, we get the space $\left(\mathscr{H}(U), \tau_{\omega}\right)$ of all holomorphic mappings on $U$, with the ported topology. $c_{0}^{+}$denotes the set of all nonnegative sequences of real numbers converging to 0 . The space $\left.\mathscr{P}_{\theta}{ }^{n} E\right)$ of $n$-homogeneous polynomials of $\theta$-type on $E$ is called intrinsic if the algebraic and topological definition of $\mathscr{P}_{\theta}\left({ }^{n} E\right)$ depends only on the algebraic and topological definition of $E$. If $U$ is the unit ball for an equivalent norm on $E$, we denote by \|\|$_{\theta, U}$ the norm on $\left.\mathscr{P}_{\theta}{ }^{n} E\right)$ corresponding to $U$. It will be convenient for us to use various equivalent norms on $E$, whose unit balls will often be expressed in terms of the unit ball $B_{1}$ of a fixed norm on $E$.

An $\alpha$-holomorphy type $\theta$ is a holomorphy type which satisfies the condition that for each $n \in N,\left(\mathscr{P}_{\theta}\left({ }^{n} E\right),\|\|_{\theta}\right)$ is intrinsic, and if $U$ and $V$ are the unit balls for two equivalent norms on $E$ such that $c U \subseteq V$ for $c \in R^{+}$, then

$$
c^{n}\left\|P_{n}\right\|_{\theta, U} \leqq\left\|P_{n}\right\|_{\theta, V},
$$

AMS 1970 subject classifications. Primary 46E10; Secondary 58C10, 58C20.

Key words and phrases. Holomorphy type, nuclear type, current type, holomorphic germ, infinite dimensional holomorphy.

1 Most of the results of this paper are taken from the author's doctoral dissertation, written under Professor Leopoldo Nachbin (University of Rochester, 1971). The author acknowledges financial assistance while a National Science Foundation Trainee. 
for all $n \in N$ and all $P_{n} \in \mathscr{P}_{\theta}\left({ }^{n} E\right)$. We shall assume that all holomorphy types are $\alpha$-holomorphy types.

2. Definition 1. (a) $H_{\theta}(U)$ is the set of all functions $f \in \mathscr{H}(U)$ which satisfy the following conditions:

(i) $2^{n} f(0) \in \mathscr{P}_{\theta}\left({ }^{n} E\right)$, for all $n \in N$.

(ii) For each $K \in \mathscr{K}(U)$, there is $\varepsilon>0$ such that

$$
\sum_{m=0}^{\infty}\left\|\frac{\partial^{m} f(0)}{m !}\right\|_{\theta, K+\varepsilon B_{1}}<\infty .
$$

(b) A seminorm $p$ on $H_{\theta}(U)$ is $K$-ported for some $K \in \mathscr{K}(U)$ if for all $\varepsilon>0$, there is $c(\varepsilon)>0$ such that, for all $f \in H_{\theta}(U)$,

$$
p(f) \leqq c(\varepsilon) \sum_{m=0}^{\infty}\left\|\frac{\partial^{m} f(0)}{m !}\right\|_{\theta, K+\varepsilon B_{1}}
$$

The topology $T_{\theta}$ on $H_{\theta}(U)$ is the locally convex topology generated by all seminorms $p$ on $H_{\theta}(U)$ which are $K$-ported for some $K \in \mathscr{K}(U)$.

THEOREM 1. (a) Let $f=\sum_{n=0}^{\infty} \partial^{n} f(0) / n$ ! be a holomorphic function on $U$, and $\partial^{n} f(0) \in \mathscr{P}_{\theta}\left({ }^{n} E\right)$ for all $n \in N$. Then, the following conditions are equivalent:

(i) $f \in H_{\theta}(U)$.

(ii) For all $K \in \mathscr{K}(U)$ and for all $\left\{\alpha_{n}\right\} \in c_{0}^{+}$,

$$
\sum_{n=0}^{\infty}\left\|\frac{\partial^{n} f(0)}{n !}\right\|_{\theta, K+\alpha_{n} B_{1}}<\infty .
$$

(iii) For all $K \in \mathscr{K}(U)$ and for all $\left\{\alpha_{n}\right\} \in c_{0}^{+}$,

$$
\lim \sup _{n}\left\|\frac{\partial^{n} f(0)}{n !}\right\|_{\theta, K+\alpha_{n} B_{1}}^{1 / n}<1 .
$$

(b) The topology $T_{\theta}$ on $H_{\theta}(U)$ is generated by all seminorms of the form

$$
p(f)=\sum_{n=0}^{\infty}\left\|\frac{\partial^{n} f(0)}{n !}\right\|_{\theta, \mathbf{K}+\alpha_{n} B_{1}},
$$

where $K \in \mathscr{K}(U)$ and $\left\{\alpha_{n}\right\} \in c_{0}^{+}$.

(c) Suppose that, for any equivalent norm on $E$ with unit ball $V$,

$$
\|P\|_{V} \leqq\|P\|_{\theta, V},
$$

for any $P \in \mathscr{P}_{\theta}\left({ }^{n} E\right), n \in N$. Then $\left(H_{\theta}(U), T_{\theta}\right)$ is complete.

3. Let $K$ be a compact, convex, balanced subset of $E$, and let $\mathscr{U}(K)$ be the family of all open, balanced neighborhoods of $K$. Let $f_{i} \in \mathscr{H}\left(U_{i}\right)$, where $U_{i} \in \mathscr{U}(K), i=1,2 . f_{1}$ is said to be equivalent to $f_{2}$ modulo $K$ if $f_{1}=f_{2}$ 
on some neighborhood of $K$ contained in $U_{1} \cap U_{2}$. Each equivalence class of functions is called a germ. The equivalence class determined by a function $f$ is denoted $[f]$, and the space of equivalence classes is called the space of holomorphic germs on $K$ and is denoted $\mathscr{H}(K)$.

Definition 2. (a) An element $[f]$ of $\mathscr{H}(K)$ is said to be of $\alpha$-holomorphy type $\theta$ if some representative of $[f]$ belongs to $H_{\theta}(U)$ for some $U \in \mathscr{U}(K)$. The space of holomorphic germs of $\alpha$-holomorphy type $\theta$ is denoted $H_{\theta}(K)$.

(b) For each $U \in \mathscr{U}(K)$, there is a natural mapping

$$
\phi_{U}:\left(H_{\theta}(U), T_{\theta}\right) \rightarrow H_{\theta}(K),
$$

taking a function $f \in H_{\theta}(U)$ into its equivalence class $[f]$ in $H_{\theta}(K)$. The topology $T_{\theta}$ on $H_{\theta}(K)$ is the inductive limit topology of the spaces $\left(H_{\theta}(U), T_{\theta}\right)$ under the mappings $\phi_{U}$, for $U \in \mathscr{U}(K)$.

THEOREM 2. (a) The topology $T_{\theta}$ on $H_{\theta}(K)$ is generated by all seminorms of the form

$$
p([f])=\sum_{n=0}^{\infty}\left\|\frac{\partial^{n} f(0)}{n !}\right\|_{\theta, K+\alpha_{n} B_{1}}
$$

where $\left\{\alpha_{n}\right\} \in c_{0}^{+}$and $f$ is any representative of $[f]$.

(b) Suppose that, for any equivalent norm on $E$ with unit ball $V$,

$$
\|P\|_{V} \leqq\|P\|_{\theta, V},
$$

for any $P \in \mathscr{P}_{\theta}\left({ }^{n} E\right), n \in N$. Then $\left(H_{\theta}(K), T_{\theta}\right)$ is complete.

(c) Suppose that, for any equivalent norm on $E$ with unit ball $V$,

$$
\|P\|_{V} \leqq\|P\|_{\theta, V},
$$

for any $P \in \mathscr{P}_{\theta}\left({ }^{n} E\right), n \in N$. Then, a subset $\mathscr{X}$ of $\left(H_{\theta}(K), T_{\theta}\right)$ is bounded if and only if for some $U \in \mathscr{U}(K)$ there is a bounded subset $X$ of $\left(H_{\theta}(U), T_{\theta}\right)$ such that $\phi_{U}(X)=\mathscr{X}$.

Remarks. (1) When $\theta$ is the current holomorphy type, $\left(H_{\theta}(U), T_{\theta}\right)$ $=\left(\mathscr{H}(U), \tau_{\omega}\right)$ and $\left(H_{\theta}(K), T_{\theta}\right)=\left(\mathscr{H}(K), \tau_{\omega}\right)$. In fact, in this case, Theorems 1 and 2 remain true without the assumption that $U$, respectively $K$, be convex (see also [2]).

(2) The condition imposed on the holomorphy type in Theorems 1 and 2 is necessary. In fact, one can give examples in which $\left(H_{\theta}(U), T_{\theta}\right)$ and $\left(H_{\theta}(K), T_{\theta}\right)$ are not complete if the condition is not satisfied.

(3) Theorem 2 extends a result of Martineau to infinite dimensions $\left[5\right.$, p. 14]. Theorem 2 can be generalized to the spaces $\left(\mathscr{H}_{\theta}(K), \tau_{\omega \theta}\right)$ of $[6]$, where $K$ is a compact set of the form $K=\bigcup_{i=1}^{n} K_{i}$, each $K_{i}$ is a compact, $\xi_{i}$-equilibrated set for some $\xi_{i} \in E$, and $\theta$ is an arbitrary holomorphy type.

(4) As in the finite dimensional case, the study of existence and uniqueness of solutions to partial differential equations on spaces of holomorphic 
mappings on open sets is quite difficult, and will be left for a later note. We also leave the study of holomorphic functions of $\alpha$-holomorphy type $\theta$ on arbitrary open subsets of a Banach space for a later note [1].

\section{REFERENCES}

1. R. M. Aron, Holomorphy type for open subsets of a Banach space, Studia Math. 45 (to appear).

2. S. B. Chae, Holomorphic germs on Banach spaces, Ann. Inst. Fourier (Grenoble) 21 (1971), fasc. 3, 107-141.

3. S. Dineen, Holomorphic functions on a Banach space, Bull. Amer. Math. Soc. 76 (1970), 883-886. MR 41 \# 4216.

4. - Holomorphy types on a Banach space, Studia Math. 39 (1971), fasc. 3, 241-288.

5. A. Martineau, Sur les fonctionelles analytiques et la transformation de Fourier-Borel, J. Analyse Math. 11 (1963), 1-164. MR 28 \# 2437.

6. L. Nachbin, Topology on spaces of holomorphic mappings, Ergebnisse der Mathematik und ihrer Grenzgebiete, Band 47, Springer-Verlag, New York, 1969. MR. 40 \#7787.

7. Convolution operators in spaces of nuclearly entire functions on a Banach space, Functional Analysis and Related Fields, edited by Felix E. Browder, Springer-Verlag, New York, 1970.

8. L. Nachbin and C. Gupta, On Malgrange's theorem for nuclearly entire functions (to appear).

DePartment of MATHEMATICS, UNIVERSity OF KENTUCKy, LeXINGTON, KeNTUCKy 40506 